\title{
Interacting dark energy in the dark $S U(2)_{R}$ model
}

\author{
Ricardo G. Landim ${ }^{\mathrm{a}, 1,2}$, Rafael J. F. Marcondes ${ }^{\mathrm{b}, 1}$, Fabrízio F. Bernardi ${ }^{\mathrm{c}, 1}$, Elcio \\ Abdalla $^{\mathrm{d}, 1}$ \\ ${ }^{1}$ Instituto de Física, Universidade de São Paulo \\ Rua do Matão, 1371, Butantã, CEP 05508-090, São Paulo, SP, Brazil \\ ${ }^{2}$ Departamento de Física e Astronomia, \\ Faculdade de Ciências da Universidade do Porto, \\ Rua do Campo Alegre 687, 4169-007, Porto, Portugal
}

Received: date / Accepted: date

\begin{abstract}
We explore the cosmological implications of the interactions among the dark particles in the dark $S U(2)_{R}$ model. It turns out that the relevant interaction is between dark energy and dark matter, through a decay process. With respect to the standard $\Lambda \mathrm{CDM}$ model, it changes only the background equations. We note that the observational aspects of the model are dominated by degeneracies between the parameters that describe the process. Thus, only the usual $\Lambda \mathrm{CDM}$ parameters, such as the Hubble expansion rate and the dark energy density parameter (interpreted as the combination of the densities of the dark energy doublet) could be constrained by observations at this moment.
\end{abstract}

\section{Introduction}

Understanding the nature of dark energy and dark matter is a puzzling challenge that has motivated physicists to develop huge observational programs. This is one of the biggest concerns in modern cosmology. The simplest dark energy candidate is a cosmological constant, in agreement with the Planck satellite results [1]. Such attempt, however, suffers from a huge discrepancy of 120 orders of magnitude between a theoretical prediction and the observed data [2]. The origin of such a constant is still an open issue which motivates physicists to look into more sophisticated models. The plethora of dark energy candidates include scalar fields [3-14], vector fields [15-21], holographic dark energy [2239], models of false vacuum decay [40-45], modifications of gravity and different kinds of cosmological fluids [4648]. In addition, the two components of the dark sector may interact with each other [14, 25-28, 44, 48-68], since their

\footnotetext{
arlandim@if.usp.br

brafaelmarcondes@usp.br

cbernardiff@gmail.com

deabdalla@if.usp.br
}

densities are comparable and the interaction can eventually alleviate the coincidence problem $[69,70]$.

Much closer to the Standard Model (SM) of particle physics are models based on gauge groups which aim to take dark matter into account. Those with $S U(2)_{R}$ symmetry, for instance, are known in the literature as extensions of the SM, in the so-called left-right symmetric models [71-77], where, recently, dark matter has been considered as well [78-88], but with no attempt to insert dark energy in it.

The dark $S U(2)_{R}$ model was built to have the two elements of the dark sector [89] and it is similar to the wellknown model of weak interactions. In principle, the hidden sector interacts with the SM particles only through gravity. Dark energy is interpreted as a scalar field whose potential is a sum of even self-interactions up to order six. The scalar field is at the local minimum of the potential, and such false vacuum might decay into the true one through the barrier penetration. However, in order to explain the current cosmic acceleration, the false vacuum should be long-lived (with a life time of the order of the age of the universe, as shown in [89]) and therefore the scalar field behaves as a cosmological constant. On the other hand, it differs from the latter due to the presence of interactions among the dark particles.

In this work we explore the interactions among the dark particles from the cosmological point of view. The relevant interaction is among dark energy and dark matter, through the decay process calculated in [89]. It turns out that the coupling changes only the background equations, since the dark energy perturbation decreases faster than radiation. The paper is organized in the following manner. Sect. 2 presents the dark $S U(2)_{R}$ model, introduced in [89]. In Sect. 3 we derive its cosmological equations and discuss the outcome of confronting it with observational data from the standard cosmic probes. Sect. 4 is reserved for conclusions. 


\section{The dark $S U(2)_{R}$ model}

In the dark $S U(2)_{R}$ model [89], dark energy and dark matter are doublets under $S U(2)_{R}$ and singlets under any other symmetry. The model contains a dark matter candidate $\psi$, a dark matter neutrino $v_{d}$ (which can be much lighter than $\psi$ ), and the dark energy doublet $\varphi$, with $\varphi^{0}$ and $\varphi^{+}$, the latter being the heaviest particle by definition. The scalar potential for the dark energy doublet is

$V(\Phi)=\frac{m^{2}}{2} \Phi^{\dagger} \Phi-\frac{\lambda}{4}\left(\Phi^{\dagger} \Phi\right)^{2}+\frac{g^{\prime}}{\Lambda^{2}}\left(\Phi^{\dagger} \Phi\right)^{3}$,

where $\lambda$ and $g^{\prime}$ are positive constants and $\Lambda$ is the cutoff scale. There are also terms which involve couplings with the dark Higgs so that after the spontaneous symmetry breaking the physical mass of the dark energy doublet is no longer the same, but $m_{\varphi^{0}}$ and $m_{\varphi^{+}}$.

The dimension six interaction term can be split into

$\frac{g^{\prime}}{\Lambda^{2}}\left(\Phi^{\dagger} \Phi\right)^{3}=\left[\frac{\lambda}{32 m_{i}^{2}}+\frac{g}{\Lambda^{2}}\right]\left(\Phi^{\dagger} \Phi\right)^{3}$,

where $g^{\prime}=\frac{\lambda^{2}}{32 m_{i}^{2}} \Lambda^{2}-g$ and $i$ stands for $\varphi^{0}$ or $\varphi^{+}$.

The mass term, the quartic interaction and the first term of the dimension six operator can be grouped as a perfect square, $\left[\frac{m_{i} \varphi^{i}}{\sqrt{2}}-\frac{\lambda\left(\varphi^{i}\right)^{3}}{\sqrt{32} m_{i}}\right]^{2}$, which has an absolute minimum at $V\left(\varphi^{i}\right)=0$. The extra term $g \Lambda^{-2}\left(\varphi^{i}\right)^{6}$ brings the minimum $V( \pm 2 m / \sqrt{\lambda})$ downwards, thus the difference between the true vacuum and the false one, given by $V(0)-V\left( \pm 2 m_{i} / \sqrt{\lambda}\right)=$ $64 m_{i}^{6} \lambda^{-3} g \Lambda^{-2}$, is the observed vacuum energy. A gravityinduced term $\left(g M_{p l}^{-2}\left(\varphi^{i}\right)^{6}\right)$, which may parametrizes a graviton loop contribution [90], is a natural option since we are dealing with gravitational effects, therefore, the reduced Planck mass is the cutoff scale. ${ }^{1}$ The mass of the scalar field should be, for instance, $\sim \mathscr{O}(\mathrm{GeV})$ for $\lambda \sim g \sim 1$ in order to explain the observed value of $10^{-47} \mathrm{GeV}^{4}$. The value of the observed vacuum energy constrains one of the three parameters, namely, $m_{i}, \lambda$, or $g$.

The interaction between the fields are given by the Lagrangian

$\mathscr{L}_{\text {int }}=g\left(W_{d \mu}^{+} J_{d W}^{+\mu}+W_{d \mu}^{-} J_{d W}^{-\mu}+Z_{d \mu}^{0} J_{d Z}^{0 \mu}\right)$,

where the currents are

$$
\begin{gathered}
J_{d W}^{+\mu}=\frac{1}{\sqrt{2}}\left[\bar{v}_{d R} \gamma^{\mu} \psi_{R}+i\left(\varphi^{0} \partial^{\mu} \bar{\varphi}^{+}-\bar{\varphi}^{+} \partial^{\mu} \varphi^{0}\right)\right] \\
J_{d W}^{-\mu}=\frac{1}{\sqrt{2}}\left[\bar{\psi}_{R} \gamma^{\mu} v_{d R}+i\left(\varphi^{+} \partial^{\mu} \bar{\varphi}^{0}-\bar{\varphi}^{0} \partial^{\mu} \varphi^{+}\right)\right] \\
J_{d Z}^{0 \mu}=\frac{1}{2}\left[\bar{v}_{d R} \gamma^{\mu} v_{d R}-\bar{\psi}_{R} \gamma^{\mu} \psi_{R}+i\left(\varphi^{+} \partial^{\mu} \bar{\varphi}^{+}-\bar{\varphi}^{+} \partial^{\mu} \varphi^{+}\right)\right. \\
\left.-i\left(\varphi^{0} \partial^{\mu} \bar{\varphi}^{0}-\bar{\varphi}^{0} \partial^{\mu} \varphi^{0}\right)\right] .
\end{gathered}
$$

The behaviour of this system is two fold. If we place the bosonic field on the (metastable) vacuum at $\varphi^{i}=0$ it might

${ }^{1}$ It is possible to get the scalar potential (1) from minimal supergravity, for instance. However, as usual in supergravity theories, we ended up with a negative cosmological constant. decay or remain there in case the height and width of the barrier is large enough. We suppose that is the case here [89]. In such a case, making a background-perturbation split of the fields, they get expanded around the vacuum and the perturbations act as quantum fields in the interactions. The bosonic field $\varphi^{+}$, if trapped by a large enough barrier, can only decay by means of the Lagrangian, Eq. (3). Notice that since the false vacuum is at $\varphi^{+}=0$, the expanded Lagrangian coincides with the original one. Therefore, in the decay of the bosonic field $\varphi^{+}$into fermions plus $\varphi^{0}$ there is no potential barriers, thus the dark energy decays into dark matter and other particles. This mechanism is the one responsible for the dark energy decay into cold (or even warm) dark matter and it is what we are going to pursue now.

From a cosmological point of view, the relevant interactions among the dark particles are the decay $\varphi^{+} \rightarrow \varphi^{0}+$ $\psi+v_{d}$ [89] and the annihilation of two scalars into two fermions. The last process, however, gives a zero cross section after expanding it in even powers of $p / m$ for a fermionic cold dark matter, while the previous (decay) process has already non relativistic contributions; generally speaking, it has more important contributions. The other annihilation processes belong to the hidden sector and do not play a major role in current observations.

\section{Model predictions}

\subsection{Background equations}

The Boltzmann equation for a process $\alpha \rightarrow a+b+c$ is given by [91]

$$
\begin{aligned}
\frac{\partial\left(a^{3} n_{\alpha}\right)}{\partial t}= & -a^{3} \int d \Pi_{\alpha} d \Pi_{a} d \Pi_{b} d \Pi_{c}\left(2 \pi^{4}\right) \times \\
& \times \delta^{4}\left(p_{\alpha}-p_{a}-p_{b}-p_{c}\right)|\mathscr{M}|_{\alpha \rightarrow a+b+c}^{2} f_{\alpha}
\end{aligned}
$$

where $d \Pi_{i} \equiv \frac{1}{(2 \pi)^{3}} \frac{d^{3} p_{i}}{2 E_{i}}$ and $f_{i}=e^{-E_{i} / k_{B} T}$ and the $a=a(t)$ is the scale factor. We neglect the factors due to Bose condensation or Fermi degeneracy. The right-hand side of Eq. (7) becomes

$$
\begin{aligned}
& \int d \Pi_{\varphi^{+}} d \Pi_{\varphi^{0}} d \Pi_{\psi} d \Pi_{v}\left(2 \pi^{4}\right) \delta^{4}\left(p_{\varphi^{+}}-p_{\varphi^{0}}-p_{\psi}-p_{v}\right) \times \\
& \quad \times|\mathscr{M}|^{2} e^{-E_{\varphi^{+}} / k_{B} T}=-\Gamma n_{\varphi^{+}}
\end{aligned}
$$

where $\Gamma$ is the integral of the scattering amplitude, which in turn does not depend on $P$. The number density is $n_{\varphi^{+}}=$ $\int e^{-E_{\varphi^{+}} / k_{B} T} \frac{d^{3} p_{\varphi^{+}}}{(2 \pi)^{3}}$. Eqs. (7) and (8) lead to the following equations for the particles in the decay process

$$
\begin{gathered}
\frac{\partial\left(a^{3} n_{\alpha}\right)}{\partial t}=-\Gamma a^{3} n_{\alpha}, \\
\frac{\partial\left(a^{3} n_{a, b, c}\right)}{\partial t}=\Gamma a^{3} n_{\alpha} .
\end{gathered}
$$


Once the field is at rest in the minimum of the potential, from Eq. (9) we see that the term $a^{3} n$ should be constant (in the absence of decay) to describe the cosmological constant, therefore the energy density for a fluid with equation of state -1 should be $\rho=a^{3} m n$, that is, a non-relativistic fluid that is not diluted as the universe expands.

The continuity equation for a cosmological fluid is obtained from the definition $\rho_{i} \equiv \int \frac{d^{3} p_{i}}{(2 \pi)^{3}} E_{i} f_{i} \approx m_{i} n_{i}$, where the last equality holds for non-relativistic fluids. Hence, the continuity equation for the $\varphi^{+}$fluid is

$\dot{\rho}_{\varphi^{+}}=-\Gamma \rho_{\varphi^{+}}$,

which has the usual exponential decay solution $\rho_{\varphi^{+}} \propto e^{-\Gamma t}$. The decay rate can be seen as part of an effective equation of state for $\varphi^{+}$, since

$\dot{\rho}_{\varphi^{+}}+3 H\left(1+w_{\text {eff }}\right) \rho_{\varphi^{+}}=0$

where $w_{\text {eff }}=-1+\Gamma / 3 H$. The second term gives rise to a kinetic contribution for the dark energy.

The other fluids $\left(\varphi^{0}, \psi\right.$, and $\left.v_{d}\right)$ have similar continuity equations, with the equations of state $w_{\varphi^{+}}=-1, w_{\psi}=0$ and either $w_{v}=0$ or $w_{v}=1 / 3$. The two particles of the dark energy doublet and the dark matter candidate are nonrelativistic, which implies that the continuity equations for the remaining fluids are

$$
\begin{aligned}
\dot{\rho}_{\varphi^{0}} & =\mu_{\varphi^{0}} \Gamma \rho_{\varphi^{+}} \\
\dot{\rho}_{\psi}+3 H \rho_{\psi} & =\mu_{\psi} \Gamma \rho_{\varphi^{+}}, \\
\dot{\rho}_{v}+3 H\left(1+w_{v}\right) \rho_{v} & =\left(1-\mu_{\varphi^{0}}-\mu_{\psi}\right) \Gamma \rho_{\varphi^{+}}
\end{aligned}
$$

where in the last equation we have used the energy conservation $E_{v}=m_{\varphi^{+}}-m_{\varphi^{0}}-m_{\psi}$, which is also evident from the energy-momentum tensor conservation, and $\mu_{\varphi^{0}}, \mu_{\psi}$ are the mass ratios $m_{\varphi^{0}} / m_{\varphi^{+}}, m_{\psi} / m_{\varphi^{+}}$, respectively. The righthand side of the continuity equations above are a leadingorder approximation since we are considering non-relativistic fluids for $\varphi^{+}, \varphi^{0}$ and $\psi$.

\subsection{Cosmological perturbations}

Once the equation of state parameters $w_{i}$ are constant for all fluids, their sound speeds are $c_{s, i}^{2}=\delta \mathscr{P}_{i} / \delta \rho_{i}=w_{i}$, where $\mathscr{P}_{i}$ is the pressure of the fluid ' $i$ '. The sound speed for a scalar field is, in turn, $c_{s, \varphi}^{2}=1$ [92]. Following the definitions of [93], in the synchronous gauge the energy conservation leads to the following equations for the dark fluids

$$
\begin{aligned}
\dot{\delta}_{\varphi^{+}}+6 H \delta_{\varphi^{+}} & =-\Gamma \delta_{\varphi^{+}} \\
\dot{\delta}_{\varphi^{0}}+6 H \delta_{\varphi^{0}} & =\mu_{\varphi^{0}} \Gamma \delta_{\varphi^{+}} \\
\dot{\delta}_{\psi}+\theta_{\psi}+\frac{\dot{h}}{2} & =\mu_{\psi} \Gamma \delta_{\varphi^{+}} \\
\dot{\delta}_{v}+\left(1+w_{v}\right)\left(\theta_{v}+\frac{\dot{h}}{2}\right) & =\left(1-\mu_{\varphi^{0}}-\mu_{\psi}\right) \Gamma \delta_{\varphi^{+}}
\end{aligned}
$$

where $\delta_{i} \equiv \delta \rho_{i} / \bar{\rho}_{i}$. The right-hand sides of the equations above follow from Eqs. (12-15). Eq. (16) has the solution $\delta_{\varphi^{+}} \propto a^{-6} e^{-\Gamma t}$, in agreement with the fact that dark energy does not cluster on sub-horizon scales [94]. Since the $\varphi^{+}$ fluid is diluted in the universe faster than radiation, the couplings in the right side of Eqs. (17-19) are negligible. As a result, $\delta \varphi^{0} \propto a^{-6}$ and the continuity equation for the dark matter perturbation is the same as in the uncoupled case.

In order to get the interacting term in the momentum conservation equations, we multiply the right-hand side of Eq. (8) by $p_{\varphi^{+}} / E_{\varphi^{+}}=p_{\varphi^{+}} / m_{\varphi^{+}}$before integrating it. The field velocity is defined as $v^{i} \equiv \frac{1}{n} \int d^{3} p \frac{p \hat{p}^{i}}{E} e^{-E / T}$, thus the Navier-Stokes equation in momentum space for $\varphi^{+}$is

$k^{2} \delta_{\varphi^{+}}=\theta_{\varphi^{+}} \Gamma$,

where $\theta \equiv i k_{j} v^{j}$. The field velocity for $\varphi^{+}$is also negligible because the left-hand side of Eq. (20) goes to zero. Thus the momentum transfer is irrelevant and the Navier-Stokes equation for dark matter is the usual one from the $\Lambda \mathrm{CDM}$ model. Therefore, the decay process changes only the background equations.

\subsection{Comparison with observations}

From the observational point of view, the two scalar fields $\varphi^{+}$and $\varphi^{0}$ have $w_{i}=-1$ and effectively behave like one "dark energy" fluid. The same happens with the two particles in the dark matter doublet in the case that the dark neutrino is non-relativistic $\left(w_{v}=0\right)$. For this doublet, the background equations (14) and (15) can be combined into

$\dot{\rho}_{\mathrm{dm}}+3 H \rho_{\mathrm{dm}}=\left(1-\mu_{\varphi^{0}}\right) \Gamma \rho_{\varphi^{+}}$.

It is then straightforward to solve numerically the background cosmology in terms of the scale factor,

$$
\begin{aligned}
\frac{d \rho_{\phi^{+}}}{d a} & =-\frac{\Gamma}{a H} \rho_{\varphi^{+}}, \\
\frac{d \rho_{\varphi^{0}}}{d a} & =\mu_{\varphi^{0}} \frac{\Gamma}{a H} \rho_{\varphi^{+}}, \\
\frac{d \rho_{\mathrm{dm}}}{d a} & =-\frac{3}{a} \rho_{\mathrm{dm}}+\left(1-\mu_{\varphi^{0}}\right) \frac{\Gamma}{a H} \rho_{\varphi^{+}},
\end{aligned}
$$

backwards in time with the current densities as "initial" conditions, together with the usual equations for the standard model fluids. Rewriting the equations in terms of the scale factor eases the numerics. A degeneracy between $\Gamma$ and the density of $\varphi^{+}$is evident from these equations. The two parameters always appear multiplied. Writing them in terms of a new "density" $\rho_{\Gamma} \equiv \Gamma \rho_{\varphi^{+}}$gives

$$
\begin{aligned}
\frac{d \rho_{\Gamma}}{d a} & =-\frac{\Gamma}{a H} \rho_{\Gamma}, \\
\frac{d \rho_{\varphi^{0}}}{d a} & =\frac{\mu_{\varphi^{0}}}{a H} \rho_{\Gamma}, \\
\frac{d \rho_{\mathrm{dm}}}{d a} & =-\frac{3}{a} \rho_{\mathrm{dm}}+\frac{1-\mu_{\varphi^{0}}}{a H} \rho_{\Gamma},
\end{aligned}
$$




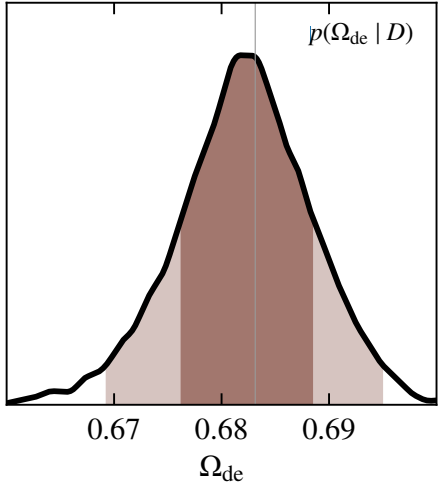

Fig. 1 Marginalized posterior probability $p$ of the derived parameter $\Omega_{\mathrm{de}} \equiv \Omega_{\varphi^{+}}+\Omega_{\varphi^{0}}$ given the data $D$ from standard cosmic probes. The shaded areas under the curve mark the $1 \sigma$ and $2 \sigma$ confidence levels; the thin grey line marks the parameter value at the best-fit (bf) point, $\Omega_{\mathrm{de}}^{(\mathrm{bf})}=0.68313$

which partially amends the problem. However, we fail to obtain constraints on the relevant parameters of the decay (now $\Gamma, \Omega_{\Gamma}, \Omega_{\mathrm{dm}}, \mu_{\varphi^{0}}$, and $\Omega_{\varphi^{0}}$ determined from the flatness condition on the sum of the density parameters) when we compare the predicted evolution with the standard cosmic probes using Markov Chain Monte Carlo (MCMC) simulations. Despite this, the derived parameter $\Omega_{\mathrm{de}} \equiv \Omega_{\varphi^{+}}+\Omega_{\varphi^{0}}$, with $\Omega_{\varphi^{+}}=\Omega_{\Gamma} / \Gamma$ is verified to mimic the standard model's dark energy with $\Omega_{\mathrm{de}}=0.68183_{-0.00564}^{+0.00668}$ at $1 \sigma$ confidence level (see Fig. 1). For this analysis, we employed observational data from the Planck Cosmic Microwave Background (CMB) distance priors [95], the Joint Light-curve Analysis (JLA) of type-Ia supernovae [96], Baryon Acoustic Oscillation (BAO) from various surveys [97-100], $H(z)$ from cosmic clocks [101, and references therein], and the local value of the Hubble constant [102]. Because the parameters $\Omega_{\Gamma}, \Gamma$ and $\mu_{\varphi^{0}}$ are expected to be small, we adopted conservative flat priors restricting them to the interval $[0,0.5]$.

The difficulties discourage any further attempt to constrain the parameters of this model in the case $w_{v}=1 / 3$, which adds two more parameters, $\Omega_{v}$ and $\mu_{\psi}$ (the dark matter doublet cannot be described as a single fluid anymore), potentially making the degeneracy even more serious.

\section{Conclusions}

In this paper we investigated the interactions among the dark particles in the dark $S U(2)_{R}$ model from a cosmological point of view. The most relevant interaction is the decay of one particle in the dark energy doublet into the other three particles in the dark energy and dark matter doublet. This process consists of a new form of interacting dark energy and it changes only the background equations. Although the comparison with data constrained very well the dark energy density parameter today, defined as the sum of the density parameters of $\varphi^{+}$and $\varphi^{0}$, the other free parameters in the process (decay rate and masses of the particles) are not constrained mainly due to the strong degeneracy between the decay rate and the density of the progenitor $\left(\varphi^{+}\right)$.

Acknowledgements This work is supported by CAPES, CNPq and FAPESP. RL would like to thank Orfeu Bertolami and the Departamento de Física e Astronomia, Faculdade de Ciências, Universidade do Porto for hosting him while the work was in progress. This work has made use of the computing facilities of the Laboratory of Astroinformatics (IAG/USP, NAT/Unicsul), whose purchase was made possible by the Brazilian agency FAPESP (grant 2009/54006-4) and the INCT-A.

\section{References}

1. P. A. R. Ade et al. Planck 2013 results. XVI. Cosmological parameters. Astron. Astrophys., 571:A16, 2014.

2. S. Weinberg. The Cosmological Constant Problem. Rev. Mod. Phys., 61:1-23, 1989.

3. P. J. E. Peebles and B. Ratra. Cosmology with a Time Variable Cosmological Constant. Astrophys. J., 325:L17, 1988.

4. B. Ratra and P. J. E. Peebles. Cosmological Consequences of a Rolling Homogeneous Scalar Field. Phys. Rev., D37:3406, 1988.

5. J. A. Frieman, C. T. Hill, and R. Watkins. Late time cosmological phase transitions. 1. Particle physics models and cosmic evolution. Phys. Rev., D46:12261238, 1992.

6. J. A. Frieman, C. T. Hill, A. Stebbins, and I. Waga. Cosmology with ultralight pseudo Nambu-Goldstone bosons. Phys. Rev. Lett., 75:2077, 1995.

7. R. R. Caldwell, R. Dave, and P. J. Steinhardt. Cosmological imprint of an energy component with general equation of state. Phys. Rev. Lett., 80:1582, 1998.

8. T. Padmanabhan. Accelerated expansion of the universe driven by tachyonic matter. Phys. Rev., D66:021301, 2002.

9. J. S. Bagla, H. K. Jassal, and T. Padmanabhan. Cosmology with tachyon field as dark energy. Phys. Rev., D67:063504, 2003.

10. C. Armendariz-Picon, V. F. Mukhanov, and P. J. Steinhardt. A Dynamical solution to the problem of a small cosmological constant and late time cosmic acceleration. Phys. Rev. Lett., 85:4438-4441, 2000.

11. P. Brax and J. Martin. Quintessence and supergravity. Phys. Lett., B468:40-45, 1999.

12. E. J. Copeland, N. J. Nunes, and F. Rosati. Quintessence models in supergravity. Phys. Rev., D62:123503, 2000. 
13. R. C. G. Landim. Cosmological tracking solution and the Super-Higgs mechanism. Eur. Phys. J., C76(8):430, 2016.

14. S. Micheletti, E. Abdalla, and B. Wang. A Field Theory Model for Dark Matter and Dark Energy in Interaction. Phys. Rev., D79:123506, 2009.

15. T. Koivisto and D. F. Mota. Vector Field Models of Inflation and Dark Energy. JCAP, 0808:021, 2008.

16. K. Bamba and S. D. Odintsov. Inflation and latetime cosmic acceleration in non-minimal Maxwell$F(R)$ gravity and the generation of large-scale magnetic fields. JCAP, 0804:024, 2008.

17. V. Emelyanov and F. R. Klinkhamer. Possible solution to the main cosmological constant problem. Phys. Rev., D85:103508, 2012.

18. V. Emelyanov and F. R. Klinkhamer. Reconsidering a higher-spin-field solution to the main cosmological constant problem. Phys. Rev., D85:063522, 2012.

19. V. Emelyanov and F. R. Klinkhamer. Vector-field model with compensated cosmological constant and radiation-dominated FRW phase. Int. J. Mod. Phys., D21:1250025, 2012.

20. S. Kouwn, P. Oh, and C.-G. Park. Massive Photon and Dark Energy. Phys. Rev., D93(8):083012, 2016.

21. R. C. G. Landim. Dynamical analysis for a vector-like dark energy. Eur. Phys. J., C76:480, 2016.

22. S. D. H. Hsu. Entropy bounds and dark energy. Phys. Lett., B594:13-16, 2004.

23. M. Li. A model of holographic dark energy. Phys. Lett., B603:1, 2004.

24. D. Pavon and W. Zimdahl. Holographic dark energy and cosmic coincidence. Phys. Lett., B628:206-210, 2005.

25. B. Wang, Y.-G. Gong, and E. Abdalla. Transition of the dark energy equation of state in an interacting holographic dark energy model. Phys. Lett., B624:141146, 2005.

26. B. Wang, Y. Gong, and E. Abdalla. Thermodynamics of an accelerated expanding universe. Phys. Rev., D74:083520, 2006.

27. B. Wang, C.-Y. Lin, and E. Abdalla. Constraints on the interacting holographic dark energy model. Phys. Lett., B637:357-361, 2006.

28. B. Wang, C.-Y. Lin, D. Pavon, and E. Abdalla. Thermodynamical description of the interaction between dark energy and dark matter. Phys. Lett., B662:1-6, 2008.

29. R. C. G. Landim. Holographic dark energy from minimal supergravity. Int. J. Mod. Phys., D25(4):1650050, 2016.

30. M. Li, X.-D. Li, S. Wang, and X. Zhang. Holographic dark energy models: A comparison from the latest observational data. JCAP, 0906:036, 2009.
31. M. Li, X.-D. Li, S. Wang, Y. Wang, and X. Zhang. Probing interaction and spatial curvature in the holographic dark energy model. JCAP, 0912:014, 2009.

32. M. Li, X.-D. Li, S. Wang, and Y. Wang. Dark Energy. Commun. Theor. Phys., 56:525-604, 2011.

33. E. N. Saridakis. Ricci-Gauss-Bonnet holographic dark energy. 2017.

34. A. Al Mamon. Reconstruction of interaction rate in holographic dark energy model with Hubble horizon as the infrared cut-off. Int. J. Mod. Phys., D26(11):1750136, 2017.

35. P. Mukherjee, A. Mukherjee, H. K. Jassal, A. Dasgupta, and N. Banerjee. Holographic dark energy: constraints on the interaction from diverse observational data sets. 2017.

36. A. Mukherjee. Reconstruction of interaction rate in Holographic dark energy. JCAP, 1611:055, 2016.

37. L. Feng and X. Zhang. Revisit of the interacting holographic dark energy model after Planck 2015. JCAP, 1608(08):072, 2016.

38. R. Herrera, W. S. Hipolito-Ricaldi, and N. Videla. Instability in interacting dark sector: An appropriate Holographic Ricci dark energy model. JCAP, 1608:065, 2016.

39. M. Forte. Holographik, the k-essential approach to interactive models with modified holographic Ricci dark energy. Eur. Phys. J., C76(12):707, 2016.

40. M. Szydłowski, A. Stachowski, and K. Urbanowski. Quantum mechanical look at the radioactive-like decay of metastable dark energy. 2017.

41. A. Stachowski, M. Szydłowski, and K. Urbanowski. Cosmological implications of the transition from the false vacuum to the true vacuum state. Eur. Phys. J., C77(6):357, 2017.

42. D. Stojkovic, G. D. Starkman, and R. Matsuo. Dark energy, the colored anti-de Sitter vacuum, and LHC phenomenology. Phys. Rev., D77:063006, 2008.

43. E. Greenwood, E. Halstead, R. Poltis, and D. Stojkovic. Dark energy, the electroweak vacua and collider phenomenology. Phys. Rev., D79:103003, 2009.

44. E. Abdalla, L. L. Graef, and B. Wang. A Model for Dark Energy decay. Phys. Lett., B726:786-790, 2013.

45. A. Shafieloo, D. K. Hazra, V. Sahni, and A. A. Starobinsky. Metastable Dark Energy with Radioactive-like Decay. 2016.

46. E. J. Copeland, M. Sami, and S. Tsujikawa. Dynamics of dark energy. Int. J. Mod. Phys., D15:1753-1936, 2006.

47. G. Dvali, G. Gabadadze, and M. Porrati. 4D Gravity on a Brane in 5D Minkowski Space. Phys. Lett. B, 485:208, 2000.

48. S. Yin, B. Wang, E. Abdalla, and C. Lin. Transition of equation of state of effective dark energy in the Dvali- 
Gabadadze-Porrati model with bulk contents. Phys. Rev., D76:124026, 2007.

49. C. Wetterich. The Cosmon model for an asymptotically vanishing time dependent cosmological 'constant'. Astron. Astrophys., 301:321-328, 1995.

50. L. Amendola. Coupled quintessence. Phys. Rev., D62:043511, 2000.

51. Z.-K. Guo and Y.-Z. Zhang. Interacting phantom energy. Phys. Rev. D., 71:023501, 2005.

52. R.-G. Cai and A. Wang. Cosmology with interaction between phantom dark energy and dark matter and the coincidence problem. JCAP, 0503:002, 2005.

53. Z.-K. Guo, R.-G. Cai, and Y.-Z. Zhang. Cosmological evolution of interacting phantom energy with dark matter. JCAP, 0505:002, 2005.

54. X.-J. Bi, B. Feng, H. Li, and X. Zhang. Cosmological evolution of interacting dark energy models with mass varying neutrinos. Phys. Rev. D., 72:123523, 2005.

55. B. Gumjudpai, T. Naskar, M. Sami, and S. Tsujikawa. Coupled dark energy: Towards a general description of the dynamics. JCAP, 0506:007, 2005.

56. A. A. Costa, X.-D. Xu, B. Wang, E. G. M. Ferreira, and E. Abdalla. Testing the Interaction between Dark Energy and Dark Matter with Planck Data. Phys. Rev., D89(10):103531, 2014.

57. E. G. M. Ferreira, J. Quintin, A. A. Costa, E. Abdalla, and B. Wang. Evidence for interacting dark energy from BOSS. Phys. Rev., D95(4):043520, 2017.

58. A. A. Costa, L. C. Olivari, and E. Abdalla. Quintessence with Yukawa Interaction. Phys. Rev., D92(10):103501, 2015.

59. A. A. Costa, X.-D. Xu, B. Wang, and E. Abdalla. Constraints on interacting dark energy models from Planck 2015 and redshift-space distortion data. JCAP, 1701(01):028, 2017.

60. R. J. F. Marcondes, R. C. G. Landim, A. A. Costa, B. Wang, and E. Abdalla. Analytic study of the effect of dark energy-dark matter interaction on the growth of structures. JCAP, 1612(12):009, 2016.

61. F. F. Bernardi and R. G. Landim. Coupled quintessence and the impossibility of an interaction: a dynamical analysis study. Eur. Phys. J., C77(5):290, 2017.

62. B. Wang, E. Abdalla, F. Atrio-Barandela, and D. Pavon. Dark Matter and Dark Energy Interactions: Theoretical Challenges, Cosmological Implications and Observational Signatures. Rep. Prog. Phys., 79(9):096901, 2016.

63. G. R. Farrar and P. J. E. Peebles. Interacting dark matter and dark energy. Astrophys. J., 604:1-11, 2004.

64. L. Santos, Wen Zhao, Elisa G. M. Ferreira, and Jerome Quintin. Constraining interacting dark energy with CMB and BAO future surveys. Phys. Rev.,
D96(10):103529, 2017.

65. W. Yang and S. Banerjee, N.and Pan. Constraining a dark matter and dark energy interaction scenario with a dynamical equation of state. Phys. Rev., D95(12):123527, 2017.

66. R. F. vom Marttens, L. Casarini, W. S. HipólitoRicaldi, and W. Zimdahl. CMB and matter power spectra with non-linear dark-sector interactions. JCAP, 1701(01):050, 2017.

67. W. Yang, S. Pan, and J. D. Barrow. Large-scale Stability and Astronomical Constraints for Coupled DarkEnergy Models. 2017.

68. X. Xu, Y.-Z. Ma, and A. Weltman. Constraining the interaction between dark sectors with future $\mathrm{HI}$ intensity mapping observations. 2017.

69. W. Zimdahl and D. Pavon. Interacting quintessence. Phys.Lett., B521:133-138, 2001.

70. L. P. Chimento, A. S. Jakubi, D. Pavon, and W. Zimdahl. Interacting quintessence solution to the coincidence problem. Phys. Rev., D67:083513, 2003.

71. J. L. Hewett and T. G. Rizzo. Low-Energy Phenomenology of Superstring Inspired E(6) Models. Phys. Rept., 183:193, 1989.

72. C. S. Aulakh, A. Melfo, and G. Senjanovic. Minimal supersymmetric left-right model. Phys. Rev., D57:4174-4178, 1998.

73. P. Duka, J. Gluza, and M. Zralek. Quantization and renormalization of the manifest left-right symmetric model of electroweak interactions. Annals Phys., 280:336-408, 2000.

74. B. A. Dobrescu and Z. Liu. $W^{\prime}$ Boson near $2 \mathrm{TeV}$ : Predictions for Run 2 of the LHC. Phys. Rev. Lett., 115(21):211802, 2015.

75. B. A. Dobrescu and P. J. Fox. Signals of a $2 \mathrm{TeV} W^{\prime}$ boson and a heavier $Z^{\prime}$ boson. JHEP, 05:047, 2016.

76. P. Ko and T. Nomura. $\mathrm{SU}(2)_{L} \times \mathrm{SU}(2)_{R}$ minimal dark matter with $2 \mathrm{TeV} W^{\prime}$. Phys. Lett., B753:612-618, 2016.

77. J. Brehmer, J. Hewett, J. Kopp, T. Rizzo, and J. Tattersall. Symmetry Restored in Dibosons at the LHC? JHEP, 10:182, 2015.

78. F. Bezrukov, H. Hettmansperger, and M. Lindner. keV sterile neutrino Dark Matter in gauge extensions of the Standard Model. Phys. Rev., D81:085032, 2010.

79. J. N. Esteves, J. C. Romao, M. Hirsch, W. Porod, F. Staub, and A. Vicente. Dark matter and LHC phenomenology in a left-right supersymmetric model. JHEP, 01:095, 2012.

80. H. An, P. S. B. Dev, Y. Cai, and R. N. Mohapatra. Sneutrino Dark Matter in Gauged Inverse Seesaw Models for Neutrinos. Phys. Rev. Lett., 108:081806, 2012. 
81. M. Nemevsek, G. Senjanovic, and Y. Zhang. Warm Dark Matter in Low Scale Left-Right Theory. JCAP, 1207:006, 2012.

82. S. Bhattacharya, E. Ma, and D. Wegman. Supersymmetric left-right model with radiative neutrino mass and multipartite dark matter. Eur. Phys. J., C74:2902, 2014.

83. J. Heeck and S. Patra. Minimal Left-Right Symmetric Dark Matter. Phys. Rev. Lett., 115(12):121804, 2015.

84. C. Garcia-Cely and J. Heeck. Phenomenology of leftright symmetric dark matter. JCAP, 1603:021, 2015.

85. A. Berlin, P. J. Fox, D. Hooper, and G. Mohlabeng. Mixed Dark Matter in Left-Right Symmetric Models. JCAP, 1606(06):016, 2016.

86. P. S. B. Dev, R. N. Mohapatra, and Y. Zhang. Heavy right-handed neutrino dark matter in left-right models. Mod. Phys. Lett., A32:1740007, 2017.

87. P. S. B. Dev, D. Kazanas, R. N. Mohapatra, V. L. Teplitz, and Y. Zhang. Heavy right-handed neutrino dark matter and PeV neutrinos at IceCube. JCAP, 1608(08):034, 2016.

88. P. S. Bhupal Dev, Rabindra N. Mohapatra, and Y. Zhang. Naturally stable right-handed neutrino dark matter. JHEP, 11:077, 2016.

89. R. G. Landim and Elcio Abdalla. Metastable dark energy. Phys. Lett. B., 764:271, 2017.

90. N. Arkani-Hamed, S. Dubovsky, L. Senatore, and G. Villadoro. (No) Eternal Inflation and Precision Higgs Physics. JHEP, 03:075, 2008.

91. E. W. Kolb and M. S. Turner. The Early Universe. Addison-Wesley, 1990. Frontiers in Physics, 69.

92. C. Gordon and W. Hu. A Low CMB quadrupole from dark energy isocurvature perturbations. Phys. Rev.,
D70:083003, 2004.

93. C.-P. Ma and E. Bertschinger. Cosmological perturbation theory in the synchronous and conformal Newtonian gauges. Astrophys. J., 455:7-25, 1995.

94. D. Duniya, D. Bertacca, and R. Maartens. Clustering of quintessence on horizon scales and its imprint on $\mathrm{HI}$ intensity mapping. JCAP, 1310:015, 2013.

95. Q.-G. Huang, K. Wang, and S. Wang. Distance priors from planck 2015 data. JCAP, 1512:022, 2015.

96. M. Betoule et al. Improved cosmological constraints from a joint analysis of the SDSS-II and SNLS supernova samples. Astron. Astrophys., 568:A22, 2014.

97. F. Beutler et al. The $6 \mathrm{dF}$ Galaxy Survey: $z \approx 0$ measurements of the growth rate and $\sigma_{8}$. MNRAS, 423:3430-3444, 2012.

98. A. J. Ross et al. The clustering of the SDSS DR7 main Galaxy sample - I. A 4 per cent distance measure at $z=0.15$. MNRAS, 449:835-847, 2015.

99. L. Anderson et al. The clustering of galaxies in the SDSS-III Baryon Oscillation Spectroscopic Survey: baryon acoustic oscillations in the Data Releases 10 and 11 Galaxy samples. MNRAS, 441:24-62, 2014.

100. A. Font-Ribera et al. Quasar-Lyman $\alpha$ forest crosscorrelation from BOSS DR11: Baryon Acoustic Oscillations. JCAP, 2014:027, 2014.

101. M. Moresco et al. A $6 \%$ measurement of the hubble parameter at $z \sim 0.45$ : direct evidence of the epoch of cosmic re-acceleration. JCAP, 1605:014, 2016.

102. A. G. Riess et al. A $2.4 \%$ determination of the local value of the Hubble constant. Astrophy. J., 826:56, 2016. 